Anti-IgE-Therapie bei Wärmeurtikaria wirksam

Eine Wärmeurtikaria ist selten, kontrollierte Therapiestudien fehlen. Ein individueller Heilversuch mit Omalizumab kann bei einer therapierefraktären Wärmeurtikaria erfolgreich sein, wie eine Kasuistik zeigt.

Ein 42-jähriger Patient litt mehrmals täglich unter oft generalisierten Wärmeurtikaria-Schüben, die teilweise mit Angioödemen und Dyspnoe einhergingen und ihn bei der Berufsausübung beeinträchtigten.

Die Diagnostik erbrachte für die Urtikariaauslösung eine Temperaturschwelle von $38^{\circ} \mathrm{C} \mathrm{im} \mathrm{warmen} \mathrm{Armbad.}$ Eine Provokation mit warmer Luft sowie ein Ergometer-Belastungstest waren ebenfalls positiv. Das Gesamt-IgE lag bei $224 \mathrm{kU} / \mathrm{l}$, spezifisches IgE war im Sx1 (häufigste Aeroallergene) nicht nachweisbar.

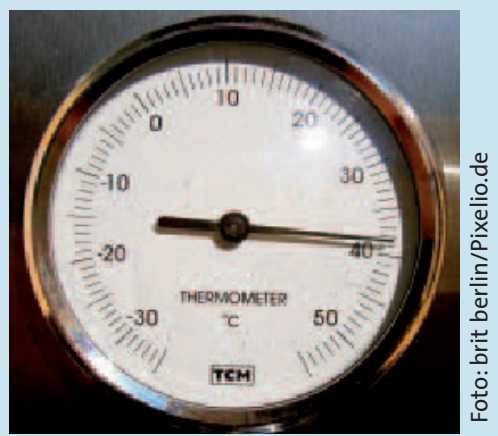

Ab $38^{\circ} \mathrm{C}$ entwickelte der Patient teils generalisierte Schübe einer Wärmeurtikaria.

Therapieversuche mit diversen Antihistaminika, Montelukast, oralen Glukokortikoiden, Cyclosporin A sowie Chloroquin führten zu keiner Besserung. Erst der Anti-IgE-Antikörper Omalizumab, zusätzlich zu Chloroquin in 14-tägigen Abständen gegeben, brachte Beschwerdefreiheit, berichtete Dr. Ute Hanne Schmidt von der Medizinischen Hochschule Hannover.

Schmidt U H, Kapp A, Wedi B. Erfolgreiche Therapie einer schweren und therapierefraktären Wärmeurtikaria mit Omalizumab. 20. Mainzer AllergieWorkshop, Mainz, 7./8. März 2008

\section{Sojabohnenallergen entdeckt}

Bislang sind die Allergene der Sojabohne noch wenig erforscht. Mit dem Nachweis von IgE-reaktiven sequenziellen Epitopen konnte $\beta$ Conglycinin jetzt als Majorallergen bestätigt werden.
S: ojaprodukte zählen wie Erdnüsse zu den häufigsten allergenen Nahrungsmitteln. Während die Allergene der Erdnuss jedoch gut charakterisiert sind, liegen zu den Sojaallergenen wenige $\mathrm{Da}$ ten vor.

Yvonne Kühne aus Langen untersuchte gemeinsam mit Kollegen das allergene Potenzial des Speicherproteins $\beta$-Conglycinin aus der Sojabohne. Dessen IgE-reaktive sequenzielle Epitope sollten identifiziert und mit denen des homologen Hauptallergens Ara h 1 der Erdnuss verglichen werden. Dazu wurden die Seren von 59 Patienten, davon 23 Kinder, analysiert, die in der Vorgeschichte oder bei Provokationstests auf Soja allergische, in sieben Fällen sogar anaphylaktische Reaktionen entwickelt hatten.

Bei mehr als der Hälfte der Patienten war aus der Vorgeschichte eine Erdnussallergie bekannt. Fast jeder zweite Sojaallergiker, über drei Viertel der Kinder und über zwei Drittel der Subgruppe mit Anaphylaxie wiesen spezifisches IgE gegen $\beta$-Conglycinin auf. Somit ist $\beta$-Conglycinin insbesondere für die Gruppe mit Anaphylaxie und für Kinder mit Soja- bohnenallergie ein bedeutsames Majorallergen, folgerte Kühne.

Bei den 26 Allergikern mit spezifischem IgE gegen $\beta$-Conglycinin wurde eine neue Methode des Epitopmapping durchgeführt, die mit sehr geringen Serenmengen auskommt und das Screening einer größeren Patientenzahl in kurzer Zeit ermöglicht. Zwei Drittel der untersuchten Seren wiesen spezifisches $\mathrm{IgE}$ gegen sequenzielle Epitope von $\beta$ Conglycinin und deutlich häufiger spezifisches IgE gegen sequenzielle Epitope von Ara h 1 auf. Ein Vergleich der IgEBindungsmuster im Epitopmapping zeigte, dass die IgE-Epitope in sequenziellen Regionen liegen, die bei beiden Allergenen konserviert sind und zusammenhängende IgE-reaktive Bereiche auf der Moleküloberfläche bilden. abd

Kühne Y, Ballmer-Weber B, Niggemann B, Scibilia J, Wackermann O, Reese G, Vieths $\mathbf{S}$, Holzhauser T. $\beta$-Conglycinin aus der Sojabohne: Allergenes Potenzial und sequenzielle Epitope. 20. Mainzer Allergie-Workshop, Mainz, 7./8. März 2008

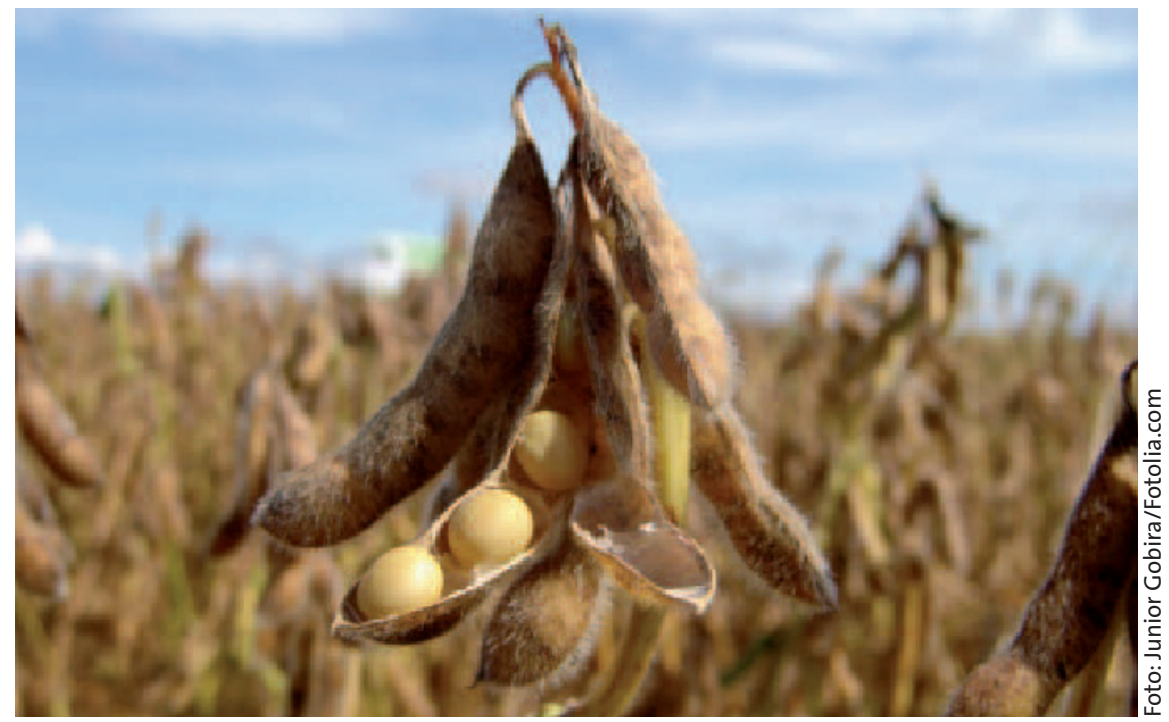

Die Sojabohne zeigt eine Epitopverwandtschaft mit der Erdnuss. 